ARTICULO

Revista Derecho - Año 2 edición 3: 107 - 120

Web: http://www.revistaderecho.pe E-mail: editorial@revistaderecho.pe

ISSN 2313-6944

\title{
IMPORTANCIA DE LA SUBSIDIARIDAD DEL ESTADO A TRAVÉS DE EMPRESAS PÚBLICAS DE DISTRIBUCIÓN DE ENERGÍA ELÉCTRICA DEL PERÚ. ANÁLISIS COMPARATIVO FINANCIERO Y SOCIAL CON LA EMPRESA PRIVADA
}

\author{
Javier Socrates Pineda Ancco*
}

INFORMACIÓN DEL ARTICULO

Art. Recibido: 20/01/16

Art. Aceptado: 07/06/16

Art. Publicado: $18 / 12 / 18$

PALABRAS CLAVE:

Distribución de energía

Subsidiariedad

Gestión

Empresa pública

\section{RESUMEN}

En la presente investigación realizamos una comparación de las empresas públicas de distribución de energía eléctrica con las empresas privadas a través de indicadores financieros y sociales para justificar la importancia de la subsidiariedad del Estado. Encontramos que las empresas privadas tienen mejores ratios financieros por la venta de energía ello porque realizan sus actividades en zonas urbanas, mientras que las empresas públicas tienen una baja rentabilidad debido a que atienden a zonas de desarrollo urbano y a zonas rurales, pero estas últimas tienen atienden a una mayor población. Esto nos demuestra que las empresas públicas tienen mejores resultados sociales pero pobres resultados financieros.

IMPORTANCE OF THE STATE'S SUBSIDIARITY THROUGH PUBLIC ELECTRICITY DISTRIBUTION COMPANIES OF PERU. FINANCIAL AND SOCIAL COMPARATIVE ANALYSIS WITH PRIVATE COMPANIES

ARTICLE INFO

Article Received: 20/01/16

Article Accepted: 07/06/16

Article Published: 18/12/18

\section{ABSTRACT}

In the present investigation, we conducted a comparison of public electric power distribution companies with private companies through financial and social indicators to justify the importance of the State's subsidiarity. We found that pri-

Filiación Institucional: Director de la Empresa Regional de Servicio Público de Electricidad de Puno Sociedad Anónima Abierta - Electro Puno S.A.A. ; Docente de la Facultad de Derecho de la Universidad Nacional del Altiplano.

Profesión: Abogado y Licenciado en Administración, Universidad Nacional del Altiplano. Grado Académico: Doctor en Derecho, Universidad Nacional del Altiplano. Correo electrónico: jvrpineda@gmail.com 
¿KEY WORDS:

Energy distribution

Subsidiarity

Management

Public company

vate companies have better financial ratios for the sale of energy because they carry out their activities in urban areas, while public companies have a low profitability due to the fact that they serve urban development areas and rural areas, but the latter have services to a larger population. This shows us that public companies have better social results but poor financial results. 


\section{INTRODUCCIÓN}

El Estado como empresario ha sido uno de los temas que más debate se ha tenido en estos últimos tiempos, debido a que desde la constitución de 1993 se cambió de un estado del bienestar a un estado regulador (Gaceta Jurídica, p.820). De acuerdo a este nuevo modelo, nos embarcamos en el modelo de la economía social de mercado, el cual establece que el Estado podrá realizar actividades empresariales subsidiariamente, es decir, que no existan en el mercado personas naturales o jurídicas de Derecho Privado que realicen la actividad empresarial que la entidad del Estado pretende desarrollar, de modo que la actividad de la empresa pública sustituya la inactividad del sector privado en un ámbito determinado. Así, la subsidiariedad significa, que la actividad del Estado como agente del mercado solo debe desarrollarse cuando no exista iniciativa privada capaz de atender determinada demanda.

Los servicios públicos de nuestro país, se ha movido en una frontera entre los público y los privado para Roberto Dromi (2005, p.62) como consecuencia de la existencia de organizaciones públicas y privadas que comparten espacios de poder, la delimitación entre lo público y lo privado varía de acuerdo con las necesidades temporales. No hay criterios científicos exactos para determinar este perímetro. Las coyunturas políticas, económicas y sociales de cada Estado condicionan de alguna manera esos límites, los cuales fueron, son y serán variables en cada circunstancia.
Por ejemplo en el sector agua potable y saneamiento, se organizó el sector como una corporación nacional en los años 60's y luego se escindió de acuerdo a la distribución territorial, para luego ser encargadas a las municipalidades Provinciales bajo la denominación de sociedades anónimas de derecho público teniendo como último desarrollo el intento de concesionar a empresa privadas el servicio como es el caso de Atusa en Tumbes. El sector telecomunicaciones, hasta antes de la Constitución de 1993, se tenía una organización pública siendo la Empresa Nacional de Telecomunicaciones del Perú la que brindaba el este servicio, luego fue privatizada a empresas españolas.

En el sector distribución de energía, del que se ocupará este documento, inicio sus actividades con iniciativa privada en 1955, la Ley No 12378 reguló los mecanismos de participación privada en este sector, pero en 1972, el Gobierno Militar de aquella época, decidió, nacionalizar todo el sector, y creo la Empresa Electroperú, (Empresa de Electricidad del Perú). Esta situación duró hasta el año 1992, cuando se emitió la Ley de Concesiones Eléctricas, que dividían a la empresa en cuatro sectores: generación, transmisión, distribución y comercialización. En los primeros dos se decidió que en su mayoría se otorgue concesiones a empresas privadas y solo quedaron pocas empresas públicas. En cuanto a la distribución y comercialización se decidió que en lugares fuera de la capital la actividad la realicen empresas públicas, y para la 
capital Lima, se concesione el servicio a empresas privadas.

En el año 1999 el Estado Peruano creo el Fondo Nacional de Financiamiento de la Actividad Empresarial del Estado - FONAFE, como una empresa de Derecho Público adscrita al Sector Economía y Finanzas creada por la Ley No. 27170, que fue promulgada el día 08.09.99, se publicó el día 09.09.99 y entró en vigencia el día 10.09.99, encargada de normar y dirigir la actividad empresarial del Estado. Al momento de su creación, FONAFE asumió las funciones de la desaparecida Oficina de Instituciones y Organismos del Estado.

El objetivo de este documento es realizar una comparación entre empresas públicas y privadas del sector distribución, para justificar la importancia del estado en su rol de subsidiariedad para el desarrollo de las regiones en el Perú.

\section{REVISIÓN BIBLIOGRÁFICA}

\section{Las empresas públicas}

El gran jurista Eduardo García de Enterría, nos manifiesta la importancia de la utilización de la técnica de las sociedades mercantiles a fin de auxiliar a la administración pública (2006, p.422) estas intervenciones iniciales va a poner de manifiesto la utilidad que presenta para la administración la adopción de una veste mercantil para la gestión de actividades industriales y comerciales, especialmente porque libera inmediatamente dicha gestión de las limitaciones del derecho público y porque supone acogerse a una técnicas (contables, de administración, de relación con el personal, de actuación en el mercado) que han hecho sobradamente sus pruebas de eficacia en el seno de la economía capitalista.

La naturaleza una empresa pública definida según Adolfo Céspedez (Cespedes, 2010), es híbrida, es decir, se trata de una persona jurídica pública, diferente a las entidades clásicas de la administración pública. Es constituida como sociedad anónima, con fines de interés social y a la vez comercial, que no son contradictorios entre sí, sino que en todo caso deben ser sopesados en cada decisión particular de la empresa, lo cual no sucede con las sociedades privadas.

Para Meirelles (1996, p. 327) las empresas públicas son personas jurídicas de derecho privado creadas por ley específica, con capital exclusivamente público, para realizar actividades de interés de la administración instituidora en los moldes de la iniciativa particular, pudiendo revestir cualquier forma de organización empresarial.

Cada empresa debe contar con la libertad Empresarial: Según la Sentencia del Tribunal Constitucional Peruano recaída en el expediente $\mathrm{N}^{\circ}$ 3330-2004-AA/TC, la libertad de empresa se manifiesta como el derecho de las personas a elegir libremente la actividad ocupacional o profesional que desee o prefiera desempeñar, disfrutando de su rendimiento económico y satisfacción espiritual. Ello es así, por una parte, en la medida en que la Constitución, en su artículo 59, reco- 
noce que "el Estado garantiza [...] la libertad de empresa, comercio e industria”. De otro lado, porque la libertad de empresa se incardina dentro de la libertad de trabajo, el cual, a su vez, es una manifestación del derecho fundamental al trabajo.

Según Jorge Castro (2011, p. 44) el artículo cuatro de la Ley General de Sociedades señalan que por lo general, toda sociedad se constituye al menos por dos socios, ya sea que éstos se traten de personas naturales o jurídicas. Y que no será exigibles la pluralidad de socios mínima a que se hace alusión anteriormente: cuando el único socio sea el Estado y en cualquier caso señalado expresamente por la ley.

Uno de los grandes problemas de la empresas públicas (EPE) según la OECD en su documento llamado Gobierno Corporativo en América Latina. (OECD, 2012) es que Las EPE se debaten entre la rentabilidad financiera y su participación en políticas públicas. Determinar el papel y los límites de actuación de las EPE a nivel de política pública es uno de los mayores desafíos para el desarrollo de este tipo de organizaciones.

Existen dos teorías presentados en el documento Public versus Private Ownership: The Current State of the Debate, para la explicación de una empresa pública y su necesidad, la primera establece que es necesario la competencia para poder ser eficiente, y mientras las empresas públicas no tiene este factor muchas veces no se desarrollan. Mientras que la segunda teoría menciona que en los monopolios naturales existe una ambigüedad si es necesario tener empresas públicas o empresas privadas, en el primer caso es necesario tener un regulador que pueda virtualmente establecer condiciones de competencia.

Una de las formas de analizar a las empresas es utilizando los instrumento financieros de rendimiento sobre capital empleado (ROCE) y el rendimiento sobre el capital invertido (ROIC) (Apaza, 2010, p.70,74), el rendimiento de capital invertido nos dice cuanto de utilidad ganamos de las inversiones que los accionistas han hecho en su empresa. Mientras que modelo ROIC se utiliza a menudo para determinar las capacidades de creación del valor de una empresa o de empresa de una manera intuitiva. Los altos nieles (relativo) de ROIC se consideran como prueba de una empresa fuerte y/o de una gerencia sólida de la empresa.

Actualmente como nuevas herramientas de control las empresas en nuestro país y dentro de ellas las empresas públicas están empezando a ser regidas por las normas financieras de contabilidad, según Pascual Ayala (2013, p.5) desde la creación del Consejo de Normas Internacionales de Contabilidad (IASB) que se instauró en reemplazo del comité de normas Internacionales de Contabilidad (IASC), a partir del año 2001 se ha dado mayor impulso a la implementación y aplicación de las Normas Internacional de Información Financiera (NIIF), que incluye NIC, NIIF, SIC y CINIFFF en todos los países del mundo. En 
nuestro país el Consejo Normativo de Contabilidad ha publicado una serie de resoluciones en las que se aprueban las modificaciones de algunas NIC y se decretan nuevas NIIF y que a partir del año 2013 están vigentes.

Otra herramienta actual es el Buen Gobierno Corporativo según el documento publicado por la OECD (2004) El gobierno corporativo constituye un elemento clave para aumentar la eficacia económica y potenciar el crecimiento, así como para fomentar la confianza de los inversores. El gobierno corporativo abarca toda una serie de relaciones entre el cuerpo directivo de una empresa, su Consejo, sus accionistas y otras partes interesadas. El gobierno corporativo también proporciona una estructura para el establecimiento de objetivos por parte de la empresa, y determina los medios que pueden utilizarse para alcanzar dichos objetivos y para supervisar su cumplimiento. Un buen gobierno corporativo deberá ofrecer incentivos apropiados al Consejo y al cuerpo directivo, para que se persigan objetivos que sirvan a los intereses de la sociedad y de sus accionistas, además de facilitar una supervisión eficaz.

\section{METODOLOGÍA}

La presente es una investigación cualitativa, a través de análisis dogmático y documental. Se analizaron los resultados de los congresos especializados del sector distribución de energía del año 2011 en el Perú, las ponencias de resultados de la OECD sobre Buen Gobierno Corporativo empresarial del Estado, Indicadores del Organismo Regulador del sector energía y minas en el Perú Osinergmin.

Posteriormente se realizó la revisión y recolección de información presentada la convensión del IV Cedelef, en especial los referentes a los indicadores de venta de energía, rentabilidad empresarial, el número de personas atendidas, el ambiente donde se desarrollan, el personal que disponen para prestar el servicio indicadores de perdida de energía y número de horas de interrupción de energía. De esta manera se pudo comparar las empresas públicas y las empresas privadas.

Dicho análisis consistió en realizar cuadros comparativos entre empresas públicas y privada desde los años 2006 a 2011.

Finalmente, se hizo un extensivo análisis crítico de las condiciones de las empresas públicas, justificando su labor subsidiaria, que la Constitución del Perú regula.

\section{ANÁLISIS DE RESULTADOS}

\section{Análisis comparativo económico - financiero}

En el Perú bajo el ámbito de operación del Fondo Empresarial del Estado - FONAFE se tiene $11 \mathrm{em}$ presas de distribución eléctrica de que abarcan abarca 22 de los 23 departamentos del Perú.

\section{Empresas de distribución eléctrica}

La cartera de empresas de Distribución Eléctrica establecida por 
FONAFE se encuentra dividida en dos grupos:

Distribuidoras 1, está conformado por las siguientes empresas:

- Electropuno

- electrosur

- $\quad$ electro sur este

- $\quad$ electro ucayali

- adinelsa

- $\quad$ sociedad eléctrica de arequipa seal

Distribuidoras 2, está conformado por las siguientes empresas:

- Electro oriente

- electrocentro

- electronoroeste

- electronorte

- hidrandina

- fonafe

Las principales funciones de los sectoristas de las Empresas de Distribución Eléctrica son:

- Supervisar la gestión de la cartera de empresas a su cargo desde una perspectiva corporativa.
- Identificar y priorizar iniciativas o proyectos corporativos que aporten valor a la cartera de empresas asignada, los cuales deberán plasmarse en planes de acción que involucren la participación de miembros seleccionados de la red de negocios u otros funcionarios de sus respectivas empresas.

- Generar mecanismos de integración y coordinación de los miembros de la red de negocios.

- Atender las solicitudes de información y/o requerimientos especiales realizadas por los miembros de la red de negocios.

\section{Análisis comparativo de empresas públicas y privadas en el sector dis- tribución de energía en el Perú}

Como se muestra en el cuadro $\mathrm{N}^{\circ} 1$ en referencia a la venta de energía a clientes finales expresados en GWh, se tiene que las empresas privadas venden el $80 \%$ de energía, y las empresas públicas solo utilizan el $20 \%$ de energía generada en el sector, cabe mencionar que los clientes de las empresas privadas son principalmente empresas privadas. 


\section{Cuadro 1}

Venta de energía a cliente final en período 2006-2011 expresado en (GWh)

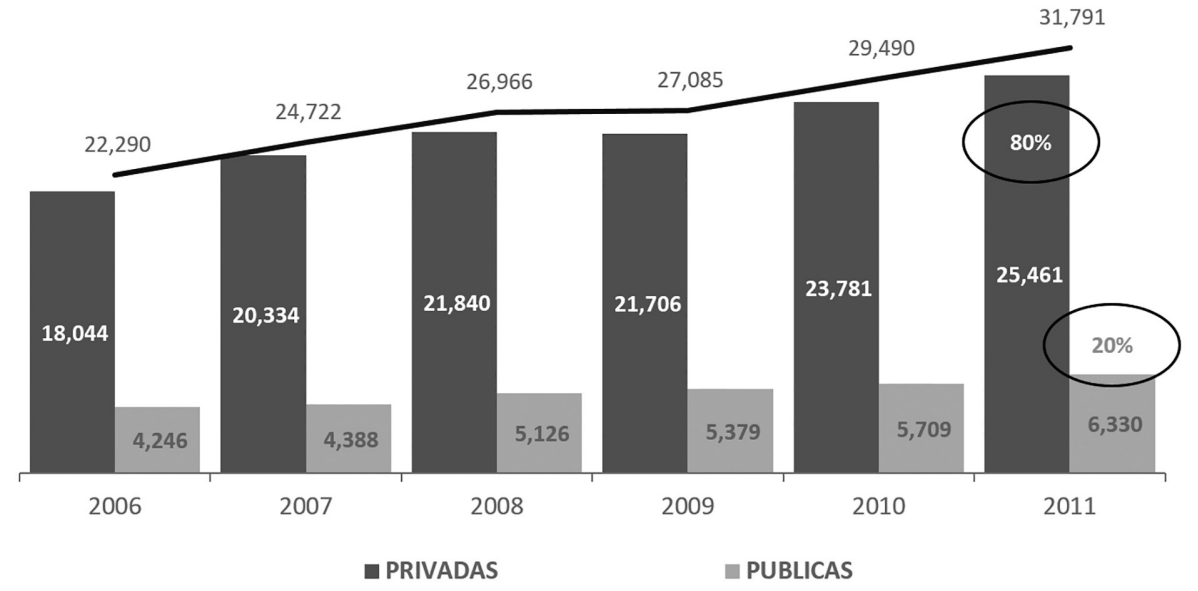

Fuente: Resumen presentado en el V CEDELEF 2012

En el cuadro $n^{\circ} 2$ encontramos, por otro lado, que el número de clientes expresado en miles, atendidos por las empresas privadas solo representan el $40 \%$ de toda la población, mientras que las empresas públicas atienden al $60 \%$ de toda la población. Por lo cual, haciendo una comparación con el cuadro anterior, se tiene que las empresas públicas si bien venden menos energía, atienden a más población en nuestro país.

\section{Cuadro 2}

Número de clientes durante período 2006-2011 (expresado en miles)

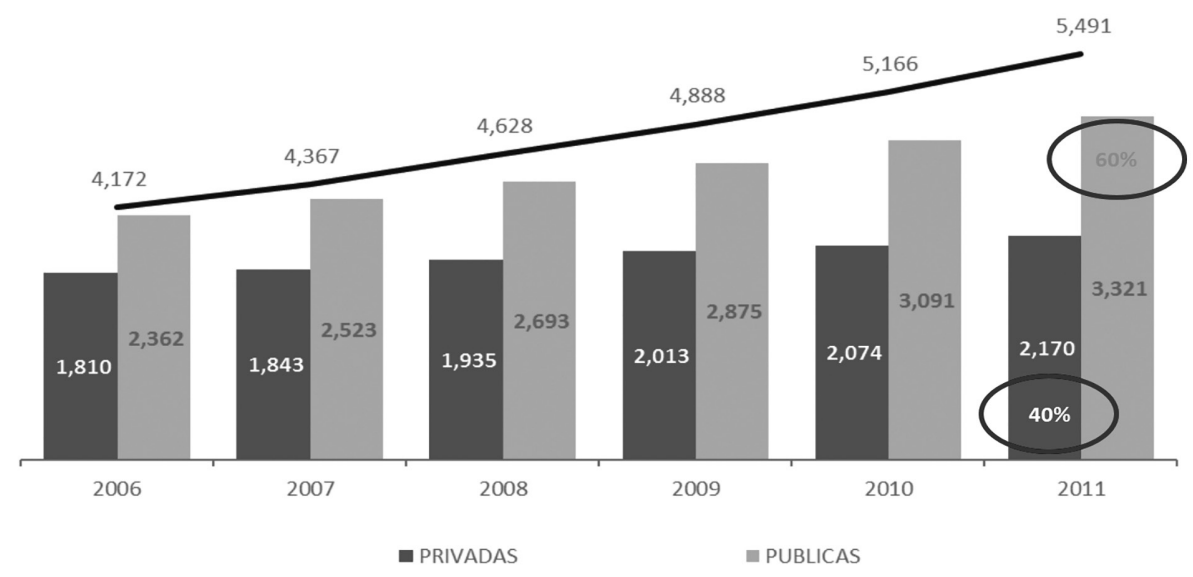

Fuente: Resumen presentado en el V CEDELEF 2012 
En el cuadro $\mathrm{N}^{\circ} 3$ se observa que las empresas del sector privado tienen una rentabilidad sobre activo ROA y rentabilidad sobre patrimonio ROE, de $17.42,22.30$ y 14.01 y 20.64 respectivamente, mientras que las empresas privadas tienen estén indicadores mucho más reducidos por ejemplo Electropuno S.A.A. tiene un ROA y ROE de 3.02 y 2.54. La justificación a esta diferencia la encontramos en que las empresas privadas atienden a solo el $4 \%$ de la población concentrado en ámbito urbano y costeño mientras las empreas públicas atienden a zonas de desarrollo urbano y clientes rurales.

\section{Cuadro 3}

Rentabilidad de las empresas públicas versus las privadas - Año 2011

(ROA y ROE expresados en porcentaje)

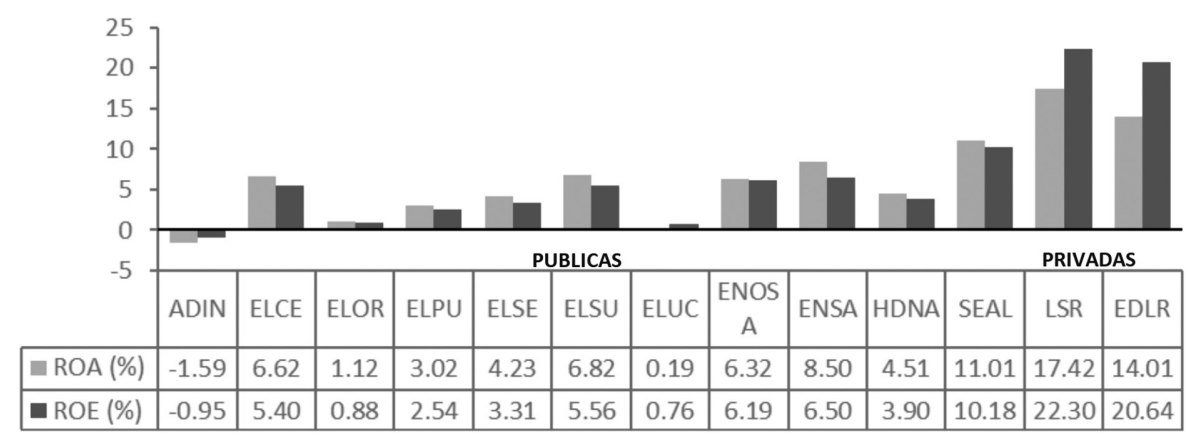

Fuente: Resumen presentado en el V CEDELEF 2012

El cuadro $\mathrm{N}^{\circ} 4$, nos muestra los resultados de número de interrupciones y la constancia del número de interrupciones, donde las empresas privadas tienen una mejor performance mientras que la mayoría de las empresas públicas tienen altos niveles de estos porcentajes.

Un tesis publicada por la Universidad Católica del Perú concluye de la siguiente manera (2010) el análisis financiero detallado de Hidrandina, la empresa más grande y representativa del grupo Distriluz, muestra que los indicadores de eficiencia — ventas por activos, pérdidas de energía, costo de ventas y gastos administrativos - no alcanzan los niveles de desempeño de las más grandes empresas del sector (Luz del Sur y Edelnor). De las zonas rural y urbana que atiende Hidrandina, solo en la segunda (sector típico 2) alcanza niveles de ventas por activos comparables con los de Edelnor y Luz del Sur. En la zona rural (sectores típicos 3, 4 y 5) esta relación es muy reducida, porque las poblaciones rurales están muy dispersas, tienen ingresos familiares y un consumo de energía muy bajos y se requieren extensas líneas y redes de distribución para atender el servicio, de donde resulta una relación ventas por activos muy reducida. Además, estos activos demandan mayores costos de operación y mantenimiento, lo que afecta la sostenibilidad del servicio. 


\section{Cuadro 4}

Dispersion del SAIDI y SAIFI

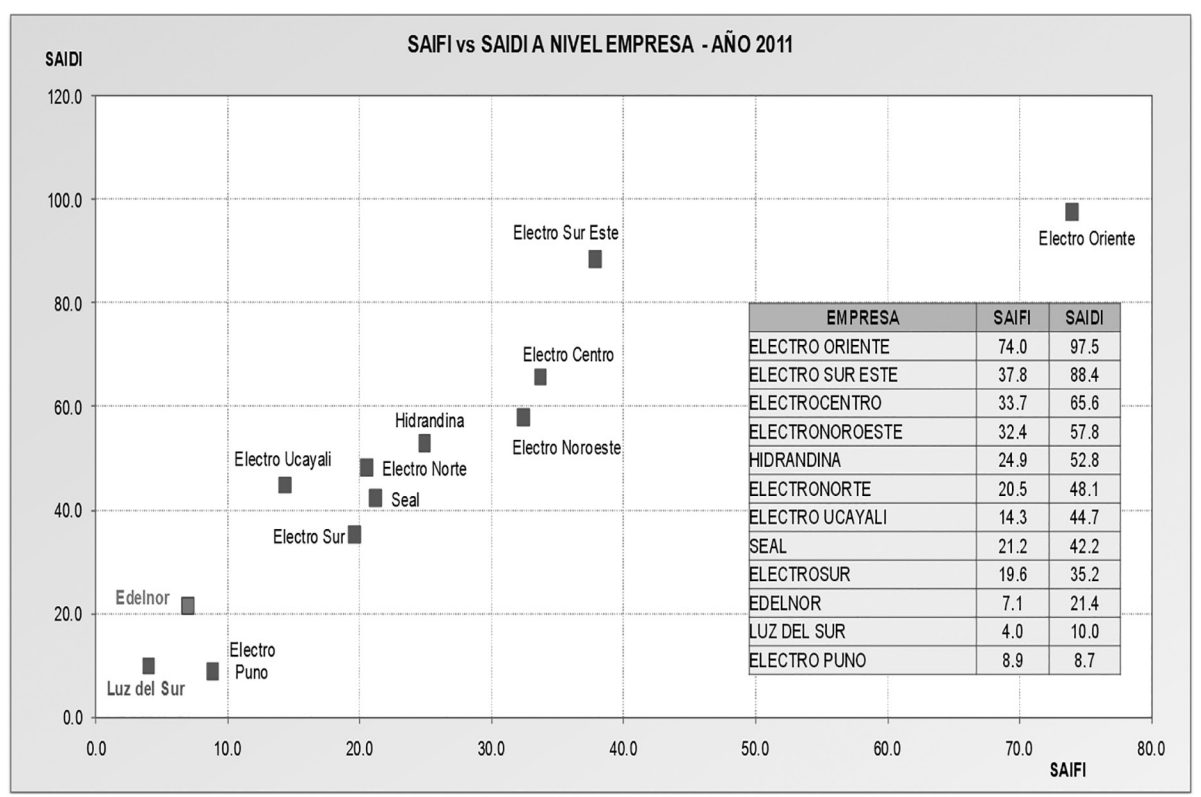

\section{Fuente: Osinergmin}

El cuadro $\mathrm{N}^{\circ} 5$ nos muestra el número de trabajadores que tienen las empresas públicas por ejemplo las empresa Electropuno S.A.A. tiene plazas en el cuadro de asignación de personal a tiene indeterminado solo
134 trabajadores para atender a trecientos mil clientes en zona alto andina. Lo que nos muestra que existiría un número muy pequeño de trabajadores para atender a tal cantidad de clientes. 


\section{Cuadro 5}

Indicadores relacionados con población laboral - Año 2011

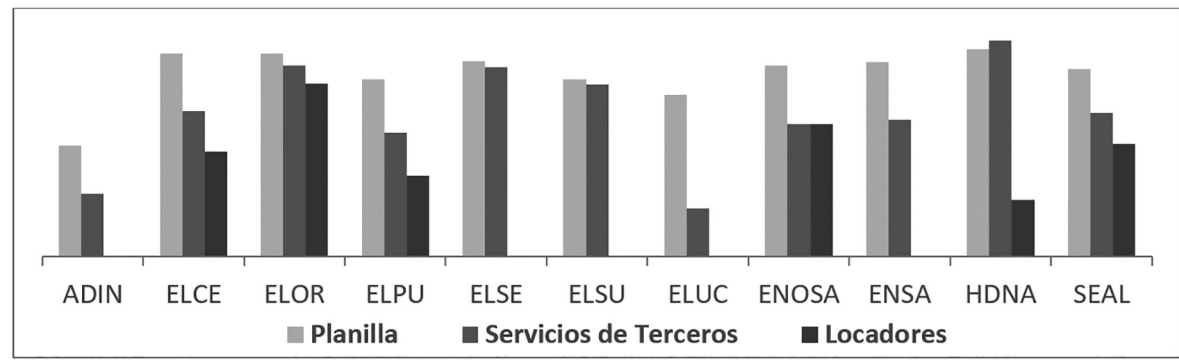

\begin{tabular}{|c|c|c|c|c|c|c|c|c|c|c|c|c|}
\hline Personal & ADIN & ELCE & ELOR & ELPU & ELSE & ELSU & ELUC & ENOSA & ENSA & HDNA & SEAL & TOTAL \\
\hline Planilla & 24 & 328 & 332 & 156 & 264 & 156 & 100 & 235 & 258 & 371 & 212 & 2,436 \\
\hline $\begin{array}{c}\text { Servicios de } \\
\text { Terceros }\end{array}$ & 6 & 63 & 234 & 34 & 221 & 135 & 4 & 44 & 49 & 475 & 61 & 1,326 \\
\hline Locadores & 0 & 20 & 138 & 10 & 0 & 1 & 1 & 44 & 0 & 5 & 25 & 244 \\
\hline CAP & 26 & 308 & 300 & 134 & 256 & 181 & 115 & 238 & 258 & 338 & 242 & 2,396 \\
\hline
\end{tabular}

Fuente: Resumen presentado en el V CEDELEF 2012

\section{Análisis de Gestión de una empresa pública en el Perú}

Todos los cuadros mostrados nos demostrarían en un primer momento que las empresas privadas tienen mejores resultados económicos y una mejor gestión empresarial, que las empresas públicas. Lo que nos lleva analizar si, al tener mejores rendimientos ellas deberían salir del ámbito público e integrar el ámbito privado, o justificar si las empresas públicas cumplen con la labor subsidiaria que exige la Constitución del Perú para su existencia.

A nivel internacional las empresas públicas, en otros países, ha logrado grandes ganancias para sus respectivas países tal es el caso de Isagen en Colombia o de Petrobras en Brasil, y a nivel mundial Statoil de Noruega, que tiene empresas en el top mundial.
Es cierto, las empresas públicas peruanas, no han podido lograr resultados con las empresas privadas debido a que si bien, tienen libertad empresarial, el estado las ha controlado con los mismos controles que cualquiera de sus entidades. Es así, que las empresas públicas para realizar compras deben regirse a lo establecido en la Ley de Contrataciones del Estado, la que establece procedimientos largos $\mathrm{y}$ muchas veces los postores alargan los procesos, mientras que las empresas privadas pueden comprar directamente sus bienes o servicios. El ex Director Ejecutivo del Fonafe, Mario Gonzales del Carpio antes de dejar su puesto manifestó en el curso 2011 Meeting of the Latin American Corporate Governance Roundtable que en el Perú, se tienen normas hechas para el sector público pero aplicadas a la actividad empresarial del estado, cosa que no ocurre en otros países 
También es importante mencionar el rol de la entidad que controla a la Administración pública peruana, La Controlaría General de la República, que al hacer sus controles a la administración, ella tiene procesos que son similares a los de las Instituciones Públicas, no haciendo la diferencia que las empresas deben maximizar utilidades económicas y brindar bienestar y no solo brindar bienestar que es la razón de las entidades públicas. En las empresas privadas los órganos de control, se preocupan con mejorar los procesos de calidad de sus empresas, mientras que la contraloría solo busca realizar controles ex post, es decir luego que ocurre el problema.

La creación del Organismo Supervisor de la Energía y la Minería Osinergmin en el Perú, ha ordenado el sector energía a través de su proceso de regulación de precios por el cual se ha podido mejorar el servicio prestado por las empresas públicas, pero desde hace pocos años su proceso regulador no se ha modificado de acuerdo a los requerimientos de nuestro mercado energético.

Es así que el proceso de fijación de precios se realiza tomando a través de estudios económicos que consideran a todas las empresas como si fueran idénticas en todos sus aspectos, cosa que no es cierto, por ejemplo en la zona alto andina empresas como Electropuno en Puno o Electro Sur Este en cusco, realizan sus labores en zonas de cambios geográficos muy pronunciados, lo que hace que el llevar energía sea más caro, pero el Osi- nergmin aún no considera estas particularidades en sus procesos.

Es de resaltar que este organismo solicita a las empresas que supervisa planes a largo plazo referidos a su desarrollo energético, donde se establece claramente los proyectos y herramientas que utilizarán, y en este punto, nos preguntamos si existe una duplicación de tareas, ello debido a que el Sistema Inversión Pública - Snip, también exige que se realice esta planificación, teniendo procedimientos engorrosos y complejos, pero que el Osinergmin también utiliza.

Legalmente, en el Derecho se conoce el principio jurídico que dice que la norma especial prevalece sobre la norma general. En este caso el Sistema de Inversión Pública es la norma general y las normas del Osinergmin son especiales, por ello, este sector debería regirse bajo las normas especiales y no regirse por las normas generales.

También es la propia organización a través del Holding de Fonafe, aún no ha logrado aprovechar la igualdad de los procesos idénticos en sus empresas. Si bien ha creado gerencias corporativas en su sede central, ellas aún no han mostrado herramientas que unifiquen los procesos de sus empresas y por tanto haga que los costos de distribución sean más bajos. En sus primeras acciones estas gerencias han lanzado los manuales de Buen Gobierno Corporativo, y han obligado a sus empresas a aceptarlos y formar reglamentos frondosos de su manejo, pero no ha realizado un plan de co- 
municación y de manejo de beneficios a sus empresas.

En una investigación realizada por Ministerio de Energía y Minas se refiere que se necesitan cera de mil millones de dólares para poder acceder a un mejor servicio de distribución de energía, ciertamente es alto monto de dinero, y el Estado no puede realizar estas inversiones en poco tiempo, sino que se ve limitado por su propia burocracia, es por ello, que una de las soluciones sería que las propias empresas públicas, puedan pedir préstamos a la banca internacional, donde se puede conseguir mejores créditos, pero para que ello ocurra es necesario que el Estado actué como aval por ser entidades públicas.

Desde nuestro punto de vista esta visión no es necesaria ya que como vimos en el análisis de acápite anterior, si se muestran índices financieros positivos en estos últimos años. Asimismo varias de estas empresas tienen un nivel de calificación crediticio positivo, y un nivel de endeudamiento muy bajo. Por ello creemos que ellas pueden financiarse sin la necesidad del Aval del Estado, pero en tanto la normativa utilizada para una entidad pública se utilice para una empresa pública ello aún está lejano.

Una de las formas para poder hacer más eficiente estas empresas públicas, es salir de los sistemas públicas, una propuesta sería aplicar algo permitido por la Ley General de Sociedad, que es el poder realizar contratos de Gerencia a personas jurídicas, así, al realizar la gestión la empresa no un gerente público sino un gerente privado, las decisiones que toma no son públicas sino privadas, por lo que, estaría fuera del sistema de gestión pública y podría utilizar herramientas del sector privado.

En este último año, las empresas han empezado a implementar el sistema de Contabilidad NIFF que ha ayudado a realizar tareas de alto nivel de complejidad en sus sistemas contables.

\section{CONCLUSIONES}

Las empresas públicas atienen da una mayor cantidad de población que las empresas privadas, pero tienen menores ratios de rentabilidad que las empresas privadas, ello por la alta dispersión de sus clientes, mientras que los clientes de las empresas privadas, se encuentran circunscritas a ámbitos muy centralizados.

Las empresas del estado para poder mejorar sus indicadores de rentabilidad deberían poder ejercer sus actividades tal cual una empresa privada, es decir, teniendo controles y formas de control, con herramientas de gestión privada, y no controlados con las herramientas del estado que están dirigidas a otro tipo de instituciones.

No es necesario que se privaticen las empresas públicas, debido a que siguen cumpliendo el rol subsidiario del Estado, al atender a población rural y en zonas de desarrollo urbano. 


\section{BIBLIOGRAFÍA}

1. Dromi, R. (2005). Derecho Administrativo. Tomo II. Lima: Gaceta Jurídica.

2. Apaza, M. (2010). Consultor Económico Financiero. Lima: Pacífico Editores.

3. Lopes, H. (19969. Direito Administrativo Brasileiro. 21va edición. Sao Paulo:Malheriros Editores LTDA.

4. García, E. (2006). Curso de Derecho Administrativo. Duodédima edición. Argentina: Thomson Civitas.

5. Robbins, S. (2000). Administración. Sexta Edición. Mexico:Prentice Hall.

6. Céspedes. A. (2010). El Estado Empresario. Lima: Ediciones Bustamente Caballero.

7. The 2012 Meeting of the Latin American Network on Corporate Governance of State-Owned Enterprises. 11-12 October, 2012 Doubletree El Pardo by Hilton Hotel. Lima, Peru.

8. La Constitución Comentada. (2005). Lima: Gaceta Jurídica.

9. Constitución Política del Perú.

10. Ley de Concesiones Eléctricas Perú.
11. VI CEDELEF (2012). Sexta convención de empresas de distribución de Fonafe.

12. Fonafe (2013). Lima. Disponible en: http://www.fonafe.gob. pe/portal?accion $=c \& \mathrm{t}=13 \& \mathrm{i}=11$ 2\&n=3\&o=104\&m=2. Accedido el 31/10/2013

13. Según la Sentencia del Tribunal Constitucional Peruano $\mathrm{N}^{\circ}$ 3330-2004-AA/TC

14. Ayala. P. (2013). NIFF Comentarios y Casuística. Lima:Editorial Pacífico.

15. Castro. J. (2011). Manual de Derecho Comercial. Lima:Juristas Editores.

16. Walshm, M. (2000). Public versus Private Ownership: The Current State of the Debate. Disponible en: http:// elibrary.worldbank.org/doi/ book/10.1596/1813-94502420. Accedido el 23/10/2013.

17. Bernal, A. (2012). Gobierno Corporativo en América Latina. Importancia para las Empresas de Propiedad Estatal. Disponible en: http://www.caf. com $/$ media $/ 73507 /$ gobiernocorporativo-importancia-empresas-estado.pdf. Accedido el 15/10/2013. 